\title{
In vivo assessment of the passive stretching response of the bicompartmental human semitendinosus muscle using shear-wave elastography
}

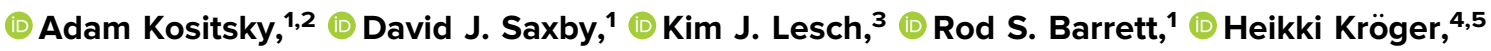 \\ (1) Olli Lahtinen, ${ }^{6,7}$ (1) Laura E. Diamond, ${ }^{1}$ (I) Rami K. Korhonen, ${ }^{2}$ and (1) Lauri Stenroth ${ }^{2}$ \\ ${ }^{1}$ Griffith Centre of Biomedical and Rehabilitation Engineering (GCORE), Menzies Health Institute Queensland, Griffith \\ University, Gold Coast, Queensland, Australia; ${ }^{2}$ Department of Applied Physics, University of Eastern Finland, Kuopio, Finland; \\ ${ }^{3}$ Sports and Exercise Medicine, Institute of Biomedicine, School of Medicine, University of Eastern Finland, Kuopio, Finland; \\ ${ }^{4}$ Department of Orthopaedics, Traumatology and Hand Surgery, Kuopio University Hospital, Kuopio, Finland; ${ }^{5}$ Kuopio \\ Musculoskeletal Research Unit (KMRU), Institute of Clinical Medicine, School of Medicine, University of Eastern Finland, \\ Kuopio, Finland; ${ }^{6}$ Diagnostic Imaging Centre, Department of Clinical Radiology, Kuopio University Hospital, Kuopio, Finland; \\ and ${ }^{7}$ Unit of Clinical Radiology, Institute of Clinical Medicine, School of Medicine, University of Eastern Finland, Kuopio, \\ Finland
}

\begin{abstract}
The semitendinosus muscle contains distinct proximal and distal compartments arranged anatomically in series but separated by a tendinous inscription, with each compartment innervated by separate nerve branches. Although extensively investigated in other mammals, compartment-specific mechanical properties within the human semitendinosus have scarcely been assessed in vivo. Experimental data obtained during muscle-tendon unit stretching (e.g., slack angle) can also be used to validate and/or improve musculoskeletal model estimates of semitendinosus muscle force. The purpose of this study was to investigate the passive stretching response of proximal and distal human semitendinosus compartments to distal (knee) joint extension. Using two-dimensional shear-wave elastography, we bilaterally obtained shear moduli of both semitendinosus compartments from 14 prone-positioned individuals at 10 knee flexion angles [from $90^{\circ}$ to $0^{\circ}$ (full extension) at $10^{\circ}$ intervals]. Passive muscle mechanical characteristics (slack angle, slack shear modulus, and the slope of the increase in shear modulus) were determined for each semitendinosus compartment by fitting a piecewise exponential model to the shear modulus-joint angle data. We found no differences between compartments or legs for slack angle, slack shear modulus, or the slope of the increase in shear modulus. We also found that the experimentally determined slack angle occurred at $\sim 15^{\circ}-80^{\circ}$ higher knee flexion angles compared with estimates from two commonly used musculoskeletal models, depending on participant and model used. Overall, these findings demonstrate that passive shear modulus-joint angle curves do not differ between proximal and distal human semitendinosus compartments and provide experimental data to improve semitendinosus force estimates derived from musculoskeletal models.
\end{abstract}

NEW \& NOTEWORTHY We conducted an elastography-based investigation of the passive stretching response of the proximal and distal compartments of the human semitendinosus muscle and found no difference in shear modulus-joint angle curves between compartments. We also found that common musculoskeletal models tend to misestimate semitendinosus slack angle, most likely due to typical model assumptions. These results provide an important step toward a better understanding of semitendinosus passive muscle mechanics and improving computational estimates of muscle force.

force; hamstrings; mechanical properties; musculoskeletal modeling; slack angle

\section{INTRODUCTION}

The semitendinosus (ST) is a fusiform hamstring muscle comprised of proximal ( $\left.\mathrm{ST}_{\text {prox }}\right)$ and distal $\left(\mathrm{ST}_{\text {dist }}\right)$ compartments separated by a band of connective tissue, termed the tendinous inscription, that commences at around one-third of muscle length and courses obliquely into the lower half of the muscle (1-3). Despite each compartment containing separate nerve branch innervations, muscle fibers in both compartments are oriented in parallel and nearly all fibers originate or terminate on the tendinous inscription, indicating an anatomically in-series arrangement of ST compartments $(1,4)$. The role of this tendinous inscription is unclear and may be an ontogenetic amalgamation of two different muscles or muscle heads $(5,6)$, a junction to connect in-series muscle fibers/fascicles (7), and/or serve to buffer forces and strains between these serially connecting muscle fibers/fascicles (8). Despite lingering questions regarding the function of the tendinous inscription, extensive investigations have revealed that $\mathrm{ST}_{\text {prox }}$ and $\mathrm{ST}_{\text {dist }}$ are mechanically in 
series in cats $(4,9,10)$. However, mechanical and material muscle properties are variable between vertebrate species (1113). For example, the maximum single fiber and fiber bundle tangent moduli are $\sim 20-25$ times greater in the frog compared with human ST muscle $(13,14)$. Furthermore, it has been established that neuromuscular properties and behavior observed in cats do not always extend to humans $(15,16)$ and thus observations made from cat ST must be empirically confirmed in human ST. Studies clearly distinguishing human $\mathrm{ST}_{\text {prox }}$ and $\mathrm{ST}_{\text {dist }}$ have been limited to measuring intramuscular electromyographic amplitudes during isometric contractions (17) and architectural and morphological measures from cadavers $(1,8,18)$ or children with and without spasticity $(19$, $20)$. As none of these previous studies directly quantified forces or stiffness within each compartment, further information is required to adequately assess the mechanical properties of human ST compartments.

Some mechanical properties of human ST compartments can be ascertained noninvasively using two-dimensional shear-wave elastography (2D-SWE), an ultrasound imaging technique quantifying Young's modulus and/or shear modulus. The strong linear relationship between muscle shear modulus obtained with 2D-SWE and force output allows shear modulus measures to be used as an index of the change in muscle force $(21,22)$. Due to technical limitations (e.g., elastography map saturation; see Refs. 23 and 24), shear modulus cannot currently be assessed in the hamstrings in vivo at maximal levels of muscle activation. Conversely, passive ST shear modulus has been assessed in several studies, during both static resting (25-27) and passive stretching (28, 29); however, no 2D-SWE studies have thoroughly examined both ST compartments in humans. Furthermore, the joint angle or musculotendinous length at which ST begins to produce passive tension (i.e., slack angle or slack length, respectively) were not quantified in previous studies, where participants' hips were highly flexed, likely because shear modulus seemingly increased immediately after stretch onset (see Fig. 5A of Ref. 28 and Fig. 2 of Ref. 29). Recently, Miyamoto et al. (27) found the resting shear modulus of hamstring muscles to be nonuniformly distributed proximodistally; however, ST compartments were not mentioned and, as measures were only obtained at four joint configurations, it remains unknown where in the range of motion passive force begins to increase in both compartments. Therefore, more detailed analyses of the passive stretching response (slack angle, slope of increase in tension, etc.) are required to better characterize and compare ST compartmental mechanical properties in humans. Furthermore, despite no between-leg asymmetry in shear modulus at two knee joint angles in uninjured individuals (26), it would be of importance to confirm that this symmetry also holds for parameters characterizing the passive stretching response to establish a baseline for potential future between-leg comparisons (e.g., previous hamstring strain injury, after anterior cruciate ligament reconstruction, etc.).

The in vivo assessment of the passive stretching response also provides important information for in silico muscle models. Most biomechanical muscle models, which are used to noninvasively estimate muscle forces due to the invasive nature of direct force measurements (30), consider passive tension to commence at the optimal fiber length (i.e., the point of maximal isometric force; see Refs. 31-33). As such, a muscle's slack length, along with its optimal fiber length, determines the joint angles over which the respective muscle produces active and passive forces in muscle models $(31,34)$. However, experimental data demonstrate that optimal length and slack length are generally not interchangeable $(12,35-39)$, causing errors in passive muscle force estimations (35). Accurate modeling of passive force generation in muscle is particularly important when studying clinical populations with spasticity (e.g., cerebral palsy, hemiplegia, etc.) $(40,41)$ or simulating surgical effects on function (e.g., tendon transfer surgeries, osteotomy, etc.) (34). Furthermore, when studying healthy populations (e.g., athletes), incorrect estimations of passive muscle force may influence estimations of total muscle force and energy cost in individual muscles during dynamic tasks that include the full range of motion (e.g., hamstring muscles during sprinting, triceps surae and quadriceps muscles during stretch-shortening cycle tasks, etc.), where large active and passive forces are present at long muscle-tendon unit lengths. In addition, common musculoskeletal models $(34,42)$ apply identical parameters for a given muscle across both limbs and represent ST as a single muscle, based on the assumption that the two compartments are also mechanically in series in humans $(43,44)$. Therefore, bilateral in vivo experimental determination of slack angle in $\mathrm{ST}_{\text {prox }}$ and $\mathrm{ST}_{\text {dist }}$ would help inform and/or validate current commonly used musculoskeletal/biomechanical models.

The aims of this study were to compare 1) passive shear modulus-joint angle curves of $\mathrm{ST}_{\text {prox }}$ and $\mathrm{ST}_{\text {dist }}$ in response to muscle-tendon unit lengthening, and 2) experimentally determined slack angles with slack angles estimated from common musculoskeletal models. Based on the results of animal studies (e.g., 4, 9, 35, 36), it was hypothesized that shear modulus-joint angle curves would not differ between $\mathrm{ST}_{\text {prox }}$ and $\mathrm{ST}_{\text {dist }}$ in either leg, and that subject-specific experimental measures of ST slack angle would differ from in silico estimates. It was also hypothesized that shear modulusjoint angle curves would not differ between legs.

\section{MATERIALS AND METHODS}

\section{Participants}

Fourteen individuals (three females, age: $28.3 \pm 4.4 \mathrm{yr}$, height: $176.1 \pm 5.4 \mathrm{~cm}$, mass: $72.0 \pm 11.4 \mathrm{~kg}$ ) volunteered to participate. Measures were obtained bilaterally; however, three participants had a prior anterior cruciate ligament reconstruction with an ipsilateral ST tendon graft on their nondominant leg. Data from the anterior cruciate ligament reconstructed legs were unused; however, data from the contralateral, uninjured legs were included as ST morphology in these legs has been shown not to differ compared with presurgical (45) and control group (46) measures. Thus, data are presented from 14 dominant and 11 nondominant legs. Potential participants were deemed ineligible if they had previous knee surgeries (other than anterior cruciate ligament reconstruction), acute muscle injuries, a body mass index $<18$ or $>35 \mathrm{~kg} \cdot \mathrm{m}^{-2}$, or any cardiorespiratory or neurological diseases. Participants were requested to refrain from strenuous exercise commencing $24 \mathrm{~h}$ before data collection. The study 
was approved by the Research Ethics Committee of the Northern Savo Hospital District (348/2020) and adhered to the Declaration of Helsinki. All participants provided written informed consent before participation.

\section{Experimental Procedure}

No warm-up was provided to avoid any confounding effect on hamstring shear moduli (25). Participants were positioned prone throughout the entire experiment. The hip was not fixed to the plinth; however, the hip hiking generally observed when participants perform active contractions was not visually observed when passively rotating through the range of motion. For preconditioning purposes, the knee joint was manually rotated slowly and passively through the range of motion five times by an investigator. Then, 2D-SWE images (see Shear-Wave Elastography Measurements section for details) were obtained at $10^{\circ}$ intervals from $90^{\circ}$ of knee flexion until full knee extension $\left(0^{\circ}\right)$ was reached, with at least $10 \mathrm{~s}$ rest given between changing knee position and subsequent image acquisition. The leg was held manually by an investigator at all angles except $0^{\circ}$, where the leg was resting along the table. Knee joint angle was verified and monitored in real-time by a calibrated electrogoniometer (ME6000, Bittium, Finland). When bilateral measurements were made, the left leg was measured first.

Surface electromyograms (ME6000, Bittium, Finland) were recorded from the semimembranosus muscle with bipolar Ag/AgCl electrodes (Ambu Blue Sensor N-00-S,
Ambu A/S, Ballerup, Denmark) placed in the direction of muscle fascicles under ultrasound guidance and with an interelectrode distance of $22 \mathrm{~mm}$. As it was not possible to simultaneously acquire elastograms and electromyograms from ST compartments, the semimembranosus muscle was chosen to represent the hamstring group as its muscle belly is more distal on the thigh (47) and thus electrodes would not interfere with positioning the ultrasound transducer over ST. Electromyography feedback was provided in realtime to both examiners and participants to ensure myoelectric silence during image acquisition.

\section{Shear-Wave Elastography Measurements}

A full description of 2D-SWE is found in detail elsewhere (21, 48, 49). Briefly, the ultrasound elastography device (LOGIQ E9, GE Healthcare, Wauwatosa, WI) calculates the tissue's Young's modulus $(E)$ for each pixel within a given region of interest by the formula:

$$
E=3 \rho c_{t}^{2}
$$

where $\rho$ is tissue density (assumed to be $1,000 \mathrm{~kg}^{*} \mathrm{~m}^{-3}$ ) and $c_{t}$ is the shear-wave speed $\left(\mathrm{m}^{*} \mathrm{~s}^{-1}\right)$. Elastography maps of $\mathrm{ST}_{\text {prox }}$ and $\mathrm{ST}_{\text {dist }}$ were obtained from images acquired using a 44mm linear transducer (9L; operating frequency: $8.4 \mathrm{MHz}$; field-of-view depth: 5 to $6 \mathrm{~cm}$ ). The elastography color map was scaled to the maximum capability of the device to be able to clearly see any potential artifacts within the map. The transducer was carefully aligned along the fascicle plane

Figure 1. Exemplary shear-wave elastography images of the proximal (left) and distal (right) compartments of semitendinosus at $80^{\circ}$ (upper) and $30^{\circ}$ (lower) of knee joint flexion. The colored rectangle within each ultrasound image is the elastography map, with the scale (Young's modulus) on the outer left of the figure. Note the similarities between ultrasound image features despite repositioning the transducer over the skin for every measurement (without the aid of indelible ink markings). The tendinous inscription is visible as the hyperechoic V-shaped structure adjacent to the elastography color maps. Examples of the measurement method using the internal software of the device are shown on the lower images.

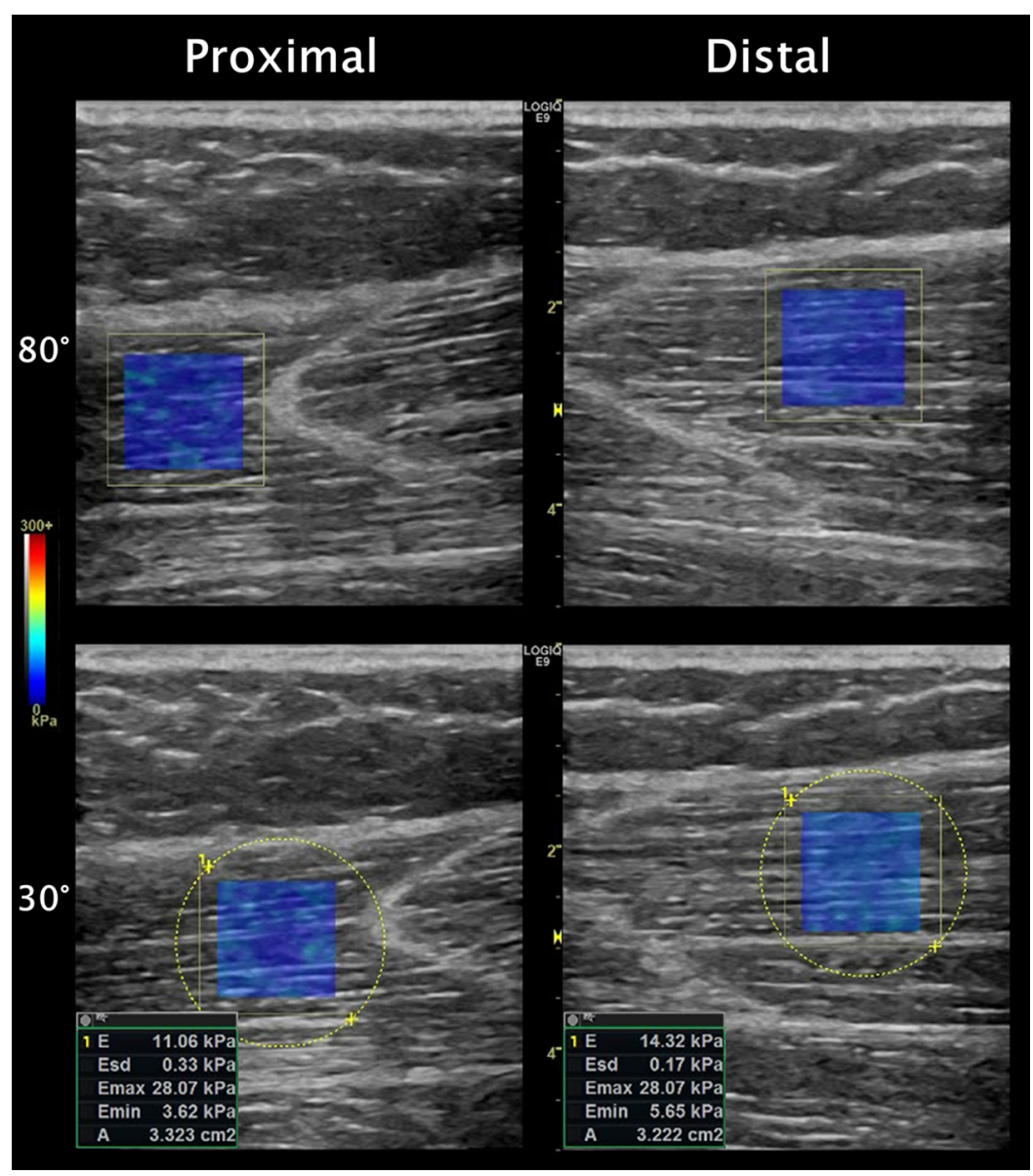


and held manually with minimal pressure over the skin (28). Transducer placement along the leg was not standardized due to interindividual variation in tendinous inscription location and substantial shifting of the ST muscle under the skin with changes in knee joint angle. Rather, at each joint angle, the transducer was placed close to the tendinous inscription, and the positioning was deemed appropriate when fascicles could be clearly seen throughout the image (Fig. 1). At any given joint angle, images were acquired from $\mathrm{ST}_{\text {prox }}$ and $\mathrm{ST}_{\text {dist }}$ in alternating fashion until three acceptable images were obtained from each compartment. Images were saved after allowing a few seconds for the elastography map to stabilize. Acoustic coupling gel was applied to prevent tissue compression, and the transducer was replaced over the skin for each image acquisition. The elastography map size was held constant at $\sim 1.15 \mathrm{~cm} \times \sim 1.15 \mathrm{~cm}$, and the same investigator (A.K.) acquired all images.

Measurements were not conducted/completed for $\mathrm{ST}_{\text {prox }}$ of both legs of one participant and the dominant leg of another participant due to poor visibility of $\mathrm{ST}_{\text {prox }}$ and/or excessive voids in the elastography map. Thus, images were obtained for $n=12$ (dominant) and $n=10$ (nondominant) for $\mathrm{ST}_{\text {prox }}$, and $n=14$ (dominant) and $n=11$ (nondominant) for $\mathrm{ST}_{\text {dist }}$. In addition, data were excluded for $\mathrm{ST}_{\text {dist }}$ at $80^{\circ}$ from one nondominant leg due to errors in the recordings, but subsequent analysis of the shear modulus-joint angle curve (see Data Analyses section) for this compartment was not affected.

\section{Data Analyses}

All elastography images were analyzed using the internal software of the ultrasound device. For each image, the mean Young's modulus value was obtained by placing a circular area around the rectangular elastography map (Fig. 1). All pixels outside or voids within the map were automatically excluded from measurements. The "Emax" output of every image was checked to ensure no pixels within the elastography map were saturated at $300 \mathrm{kPa}$ (i.e., exceeded the threshold of the device). The mean Young's modulus values were divided by three to obtain shear modulus, which, compared with Young's modulus, is a more appropriate 2D-SWE measure for anisotropic soft tissues such as muscle (21, 48, 49). The standard error of measurement of shear moduli measures was $5.9 \%-14.4 \%$. Full measurement reliability and potential effects on interpretation are described in the supplemental material (Supplemental Table S1 and Fig. S1; see https://doi.org/10.6084/m9.figshare.16837417).

A piecewise exponential model describing the passive length-tension relationship of skeletal muscle was then fitted to the data (50):

$$
\begin{array}{llrl}
G(\theta) & =G_{O} & & \text { if } \theta \leq \theta_{O} \\
G(\theta) & =G_{O}\left(e^{\alpha\left(\theta-\theta_{O}\right)}\right) & & \text { if } \theta>\theta_{O}
\end{array}
$$

where $G(\theta)$ is the shear modulus at any given knee joint angle, $G_{\mathrm{o}}$ and $\theta_{\mathrm{o}}$ are the shear modulus and knee joint angle, respectively, at which passive tension begins to develop (i.e., slack angle), and $\alpha$ is an exponential constant defining the slope of the increase in passive tension.

The values of $\theta_{\mathrm{o}}, \mathrm{G}_{\mathrm{o}}$, and $\alpha$ and were optimized using custom-made scripts in MATLAB (version R2018b, MathWorks, Natick, MA) by minimizing the least square difference between experimentally measured and modeled shear moduli using the Levenberg-Marquardt algorithm (Fig. 2). The default starting search values for the optimization were $50^{\circ}$ $\left(\theta_{0}\right), 3 \mathrm{kPa}\left(\mathrm{G}_{\mathrm{o}}\right)$, and $0.01(\alpha)$, with no boundaries. Although the initial search values would not affect the results, we elected to apply consistent values (i.e., across all legs and compartments) chosen based on initial analyses of the entire data set. The curve was fitted to the mean shear modulus value from the three images at each knee joint angle. We observed that initial decreases in shear modulus sometimes occurred at very short muscle lengths (e.g., Fig. $3 A$ of Ref. 51), which would affect the output of the piecewise model. Thus, if the shear moduli values at $90^{\circ}$ and $80^{\circ}$ did not overlap when the minimal detectable change (see Supplemental material) was accounted for, the data associated with $90^{\circ}$ was removed from the curve-fitting model ( $n=2$ legs). The coefficient of determination $\left(R^{2}\right)$ of the fit between experimental and modeled data was also calculated. The curve-fit was accepted when $\theta_{\mathrm{o}} \leq 85^{\circ}$ and $R^{2} \geq 0.85$.

The Rajagopal (42) and gait2392 (based on Ref. 34) musculoskeletal models were loaded separately in OpenSim (version 3.3, SimTK, Stanford, CA). Activation of the right ST was set at 0.01 and the hip flexion joint angle locked at $0^{\circ}$. Data from the passive fiber-force-knee joint angle curve were then extracted for every $\sim 1.21^{\circ}$ (Rajagopal) or $\sim 1.31^{\circ}$ (gait2392) of the right knee joint angle. The first joint angle where force was above zero was deemed the model-estimated $\theta_{0}$. As force-angle curves (i.e., operating range) are

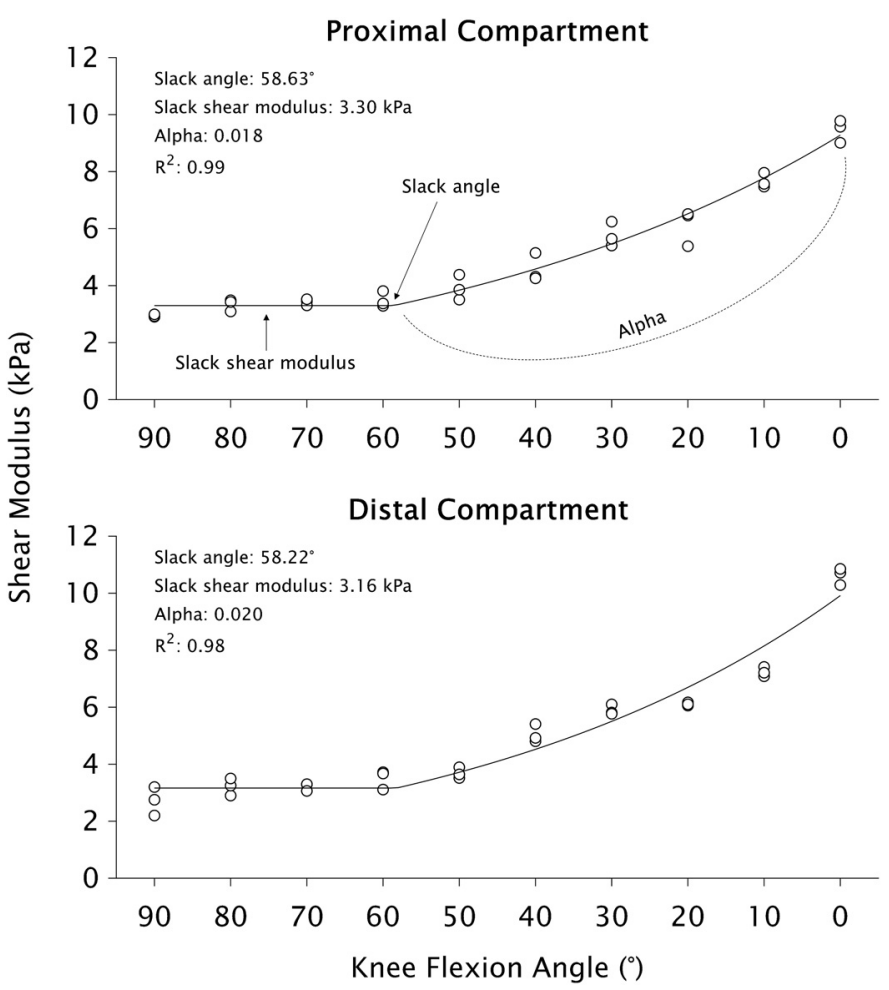

Figure 2. Representative data of the piecewise exponential model fitted to 10 knee joint angles of the proximal (upper) and distal (lower) semitendinosus compartments for one participant. Circles represent individual trial measures at each knee flexion angle $\left(0^{\circ}=\right.$ full knee extension). The solid line represents the curve fit. 
Figure 3. Boxplots of shear modulus values of proximal (unfilled) and distal (filled gray) semitendinosus compartments obtained at 10 knee flexion angles $\left(0^{\circ}=\right.$ full knee extension) of dominant (left) and nondominant (right) legs. For $\mathrm{ST}_{\text {prox }}$, data are presented for dominant $(n=12)$ and nondominant $(n=10)$ legs, and dominant $(n=14)$ and nondominant $(n=11)$ legs for $\mathrm{ST}_{\text {dist }}$ (except for $80^{\circ}$, where $n=10$; see METHODS section for details). ST, semitendinosus; $\mathrm{ST}_{\text {prox }}$, ST proximal; $\mathrm{ST}_{\text {dist }}$, ST distal.

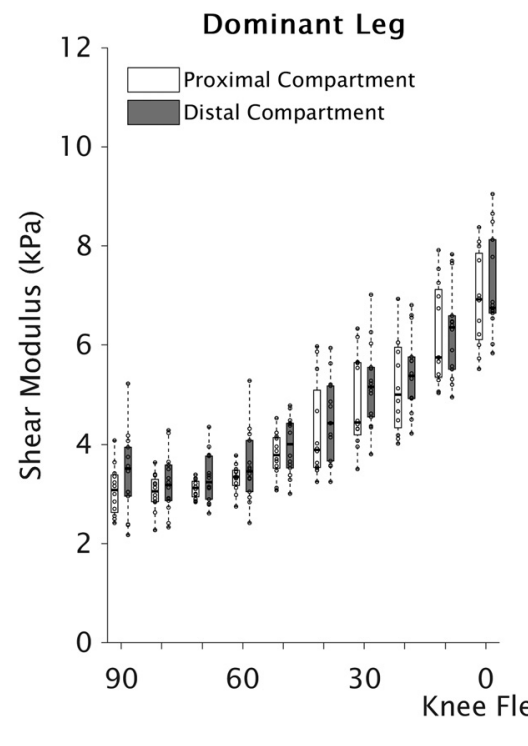

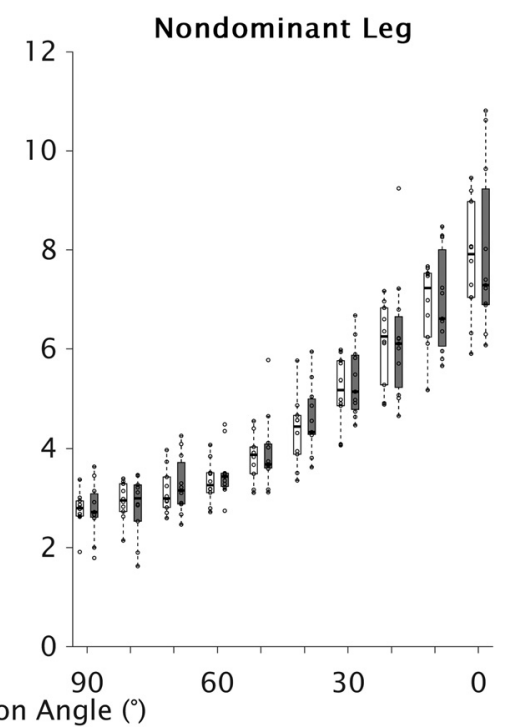

typically made generic across participants (52), only the default models were used.

\section{Statistical Analyses}

Full-factorial linear mixed models with restricted maximum likelihood estimation were performed to assess the effects of leg (dominant, nondominant) and compartment (proximal, distal) on slack angle, slack shear modulus, and $\alpha$. Leg and compartment were set as fixed factors and repeated measures (diagonal covariance structure), with intercepts for participants as a random factor. The degrees of freedom were calculated using the Satterthwaite approximation. Post hoc Bonferroni tests were conducted when significant interactions occurred. The experimentally determined slack angle (averaged across all compartments and legs for each participant) was compared with musculoskeletal model values using a one-sample $t$ test. All hypothesis testing was conducted using SPSS (version 27, SPSS Inc., Chicago, IL) with statistical significance set at $P<0.05$. Data are presented as means \pm standard deviation.

\section{RESULTS}

Shear modulus-joint angle data are summarized in Fig. 3. The piecewise model (Eq. 2) was only accepted for $37 / 47$ (79\%) tested compartments, with all individual data available in supplemental material. There were no main effects of leg or compartment for slack angle [leg: $F(1,23.96)=4.25, P=$ 0.050; compartment: $F(1,19.97)=0.57, P=0.458]$, slack shear modulus [leg: $F(1,24.51)=1.20, P=0.285$; compartment: $F(1,21.19)=2.47, P=0.131]$, or $\alpha$ [leg: $F(1,24.63)=0.48, P=$ 0.494; compartment: $F(1,20.91)=1.77, P=0.198$ ] (Fig. 4). There were also no interactions between leg and compartment for slack angle $[F(1,19.23)=0.66, P=0.426]$, slack shear modulus $[F(1,20.35)=0.40, P=0.532]$, or $\alpha[F(1,20.06)=0.95$, $P=0.341]$.

The slack angle was $24.24^{\circ}$ of knee flexion for the Rajagopal model and $2.12^{\circ}$ of knee (hyper)extension for the gait2392 model (Fig. 5). The experimentally determined slack angle $\left(62.31 \pm 8.94^{\circ}\right)$ occurred at significantly larger knee flexion angles compared with the Rajagopal $(t=15.36, P<0.001)$ and gait2392 $(t=25.99, P<0.001)$ models.

\section{DISCUSSION}

This study investigated the passive stretching response of two anatomically in-series muscle compartments, the proximal and distal compartments of the human ST muscle, in vivo using 2D-SWE. Consistent with our hypotheses, parameters from shear modulus-joint angle curves were not significantly different between ST compartments, and the experimentally observed slack angle occurred at significantly higher knee flexion angles compared with in silico estimates. These findings suggest that passive mechanical properties do not differ between the two ST compartments and provide experimental data for improving force estimates from musculoskeletal models.

\section{Semitendinosus Shear Modulus-Joint Angle Curves between Legs and Muscle Compartments}

In agreement with previous elastography studies demonstrating no difference in active (23) or passive (26) ST shear moduli between dominant and nondominant legs, no measures from the piecewise model $\left(\theta_{0}, G_{o}\right.$, or $\left.\alpha\right)$ differed between legs. Passive shear modulus-joint angle measures also did not differ between ST compartments, which is to an extent analogous with Lacourpaille et al. (53), who reported no difference in slack angle between the two heads of the biceps brachii. Conversely, Le Sant et al. (54) found slack angle to differ proximodistally within soleus, but not medial or lateral gastrocnemius, potentially due to the complex four-compartment structure of soleus (55). Despite its tendinous inscription, ST may be considered simpler than soleus as $\mathrm{ST}_{\text {prox }}$ and $\mathrm{ST}_{\text {dist }}$ are parallel-fibered and anatomically in series. Thus, given the linear relationship between the change in shear modulus and the change in passive force $(21,22)$, the lack of differences in 

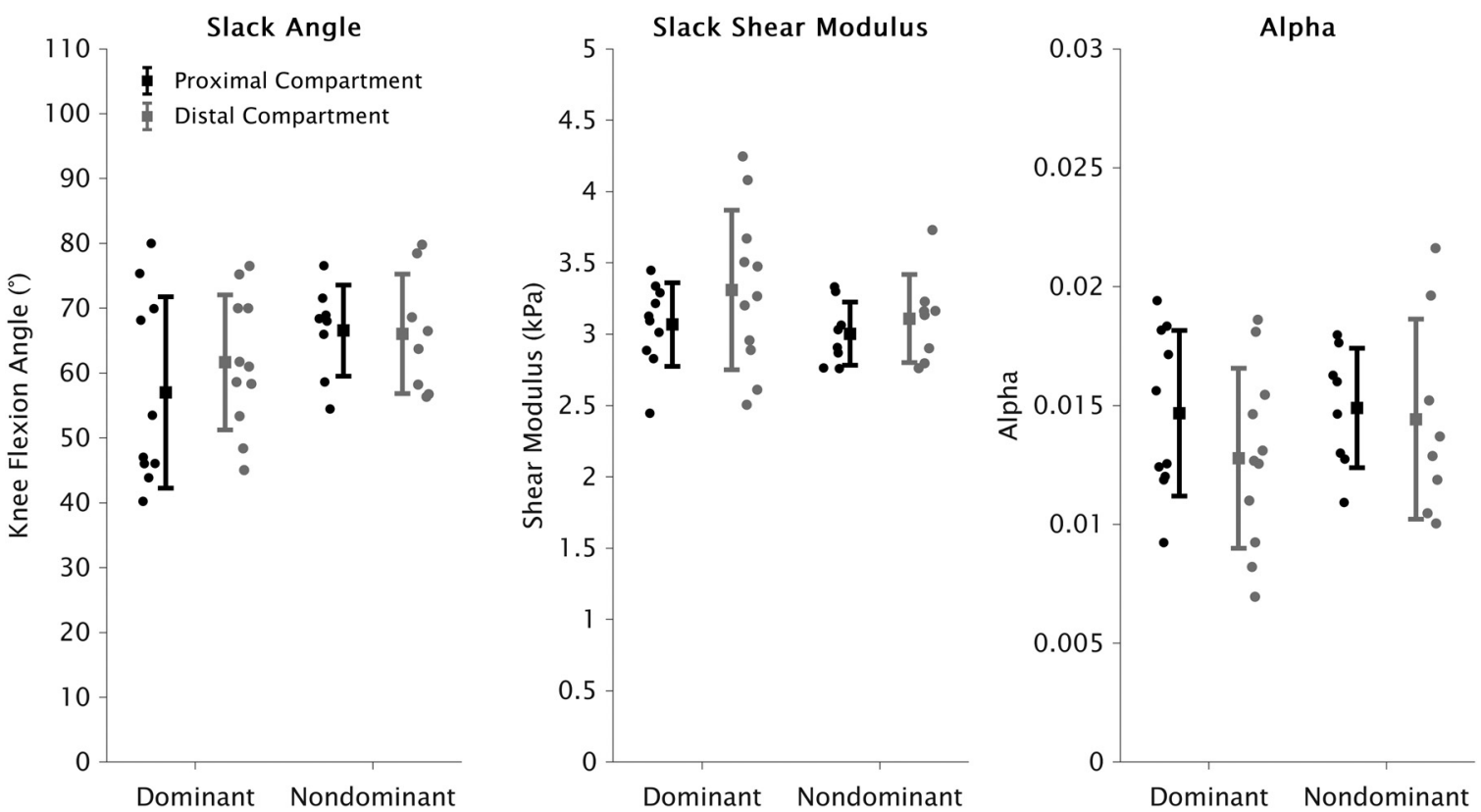

Figure 4. Means and standard deviations of slack angle (left), slack shear modulus (center), and the slope of the increase in shear modulus ( $\alpha$ ) (right) of proximal (black) and distal (gray) semitendinosus compartments of dominant and nondominant legs. Filled circles represent individual datapoints. For $\mathrm{ST}_{\text {prox }}$, data are presented for dominant $(n=10)$ and nondominant $(n=8)$ legs, and dominant $(n=11)$ and nondominant $(n=8)$ legs for ST dist. Linear mixed models found no main effects or interactions of leg and/or compartment for slack angle, slack shear modulus, or $\alpha$. ST, semitendinosus; ST prox, $S T$ proximal; ST dist, $\mathrm{ST}$ distal.

slack angle, slack shear modulus, and $\alpha$ (i.e., exponential increase in shear modulus) between $\mathrm{ST}_{\text {prox }}$ and $\mathrm{ST}_{\text {dist }}$ suggests that forces are also not different throughout the entire prone range of motion between these two anatomically in-series ST compartments, at least during passive stretching. Whether

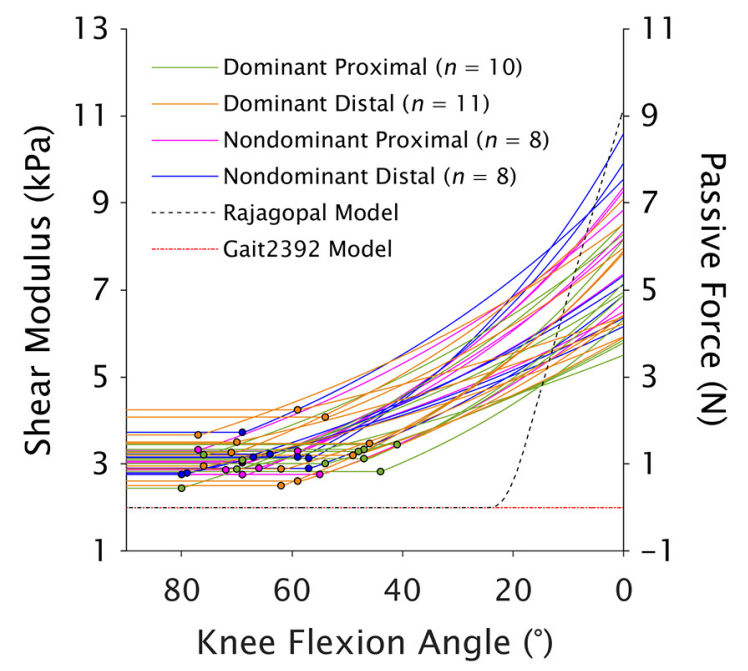

Figure 5. Comparison of experimental measures of semitendinosus slack angle with estimates from musculoskeletal models $(32,40)$, where $0^{\circ}$ of knee flexion angle represents full knee extension. Filled circles superimposed over experimental data lines represent the experimentally determined slack angle for each participant/leg/compartment. The left $y$-axis (shear modulus) corresponds with the experimental data, and the right $y$ axis (passive force) corresponds with the generic in silico data. Note there are three overlapping circles at $\sim 69^{\circ}$ of knee flexion. The slack angle determined with shear-wave elastography was significantly different $(P<0.001$; one-sample $t$ test) than the slack angle estimated from both models. forces in each compartment are independently but equally developed simultaneously or shared/balanced across compartments cannot be directly answered from the current experiment; however, some inferences may be suggested based on the results of previous studies. Stimulating the nerve branch innervating the cat $\mathrm{ST}_{\text {prox }}$ resulted in similar force recorded at the distal ST tendon compared with both $\mathrm{ST}_{\text {dist }}$ nerve branch stimulation and simultaneous stimulation of both $\mathrm{ST}_{\text {prox }}$ and $\mathrm{ST}_{\text {dist }}(4)$, and passive force development was greater in distal compared with proximal regions of the pennate rat extensor digitorum longus when the muscle was stretched distally (38). Furthermore, as the muscles directly adjacent to ST (biceps femoris long head and semimembranosus) are also biarticular hip extensors and knee flexors (43), any epimuscular myofascial force transmission affecting proximodistal distribution of muscle force should be minimal given this phenomenon is more likely to occur when adjacent muscles are displaced relative to one another $(38,56)$. Therefore, the potential for force transmission from one compartment to the other to equilibrate force across the whole human ST muscle, rather than simultaneously equal independent force development, must be strongly considered.

Although shear moduli, and thus forces, may not differ between ST compartments, the same may not hold true for stresses and strains. Unfortunately, absolute or relative length changes could not be quantified from our ultrasound images as ST fascicle length exceeds the transducer's field of view and the parallel fascicle architecture does not allow for extrapolation methods. We have, however, qualitatively observed greater movement of fascicles in $\mathrm{ST}_{\text {dist }}$ compared with $\mathrm{ST}_{\text {prox }}$ throughout the range of motion that was examined in the present study, which is consistent with previous studies reporting distal sarcomeres (57) and fascicles (58) of other 
lower limb muscles lengthen more than their proximal counterparts in response to distal joint lengthening. Considering that the physiological cross-sectional area of cadaveric $\mathrm{ST}_{\text {prox }}$ is significantly greater than $\mathrm{ST}_{\text {dist }}(18)$, larger strains in $\mathrm{ST}_{\text {dist }}$ during passive knee extension might be expected for a given shear modulus and force, as stress (force per area) would also likely be larger. Likewise, unequal strains between $\mathrm{ST}_{\text {prox }}$ and $\mathrm{ST}_{\text {dist }}$ at similar force levels have been reported in cats (4). Thus, it appears there may be a trade-off in that balancing moduli and force across two in-series compartments may result in disproportionate stresses and strains imposed on each compartment, which would have implications for musculotendinous injuries (4) but requires further investigation. Future studies that combine 2D-SWE with ultrasound strain imaging $(59,60)$ and/or microendoscopy (61) to concomitantly measure mechanical properties and length changes within each compartment are therefore recommended.

\section{In Vivo and In Silico Slack Angles}

The wide range in individual ST slack angles $\left(\sim 40^{\circ}-80^{\circ}\right)$ in the present study is comparable with interindividual variation in slack angle of other thigh muscles $(51,62)$. Interestingly, ST slack angle occurred at relatively high knee flexion angles (i.e., close to the shortest in vivo muscle length) in some participants. Recently, Kaya et al. (63) reported passive force-angle curves of spastic ST obtained intraoperatively with a buckle transducer attached to the distal tendon. Although direct comparisons are not straightforward owing to different demographic groups and methods, their data show some individuals, based on the standard deviation around the mean, developed passive ST forces even at $90^{\circ}$ of knee flexion (hip flexion $=20^{\circ}$; Fig. $3 B$ of Ref. 63). This demonstrates ST slack angle at high knee flexion angles in some healthy participants is plausible, although invasive measures would be required for verification.

Given the mechanical properties did not differ between $\mathrm{ST}_{\text {prox }}$ and $\mathrm{ST}_{\text {dist }}$, modeling the human ST as one whole muscle may be justified when estimating global muscle forces and joint contact forces in healthy, nonfatigued individuals. However, the experimentally determined ST slack angle systematically (i.e., for all compartments and legs) occurred at much deeper knee flexion angles compared with the commonly used Rajagopal (42) and gait2392 (based on Ref. 34) musculoskeletal models (difference of $\sim 15^{\circ}-80^{\circ}$, depending on participant and model used; Fig. 5). The discrepancy in slack angle may be due to errors in muscle-tendon unit length estimations in musculoskeletal models (64), but more likely originates from assumptions in biomechanical muscle models, which typically set passive force onset at optimal fiber length (31-33). Although ST sarcomere length is close to optimal length in the anatomical position $(18,43,65)$ and thus ST optimal fiber length is likely set correctly in muscle models, the main source of passive tension in whole muscles stems from extracellular matrix structures and not from the sarcomere $(66,67)$. Accordingly, experimental data have shown slack length is generally not synonymous with optimal length nor standardized across muscles (12,35-39), leading to inaccurate predictions of passive forces when assuming passive force onset at optimal fiber length (35). Although passive ST forces through the knee range of motion in the prone position are small, due to the exponential shape of the passive tension curve, total forces toward the end of the range of motion (i.e., with the hip flexed) can be severely misestimated as small length changes at long lengths cause large differences in passive forces (68). Given the discrepancy between experimental and modeled ST slack angle, this assumption may have influenced estimations of force distribution within the hamstrings during, for example, terminal swing phase during running $(69,70)$ by incorrectly allocating individual muscle forces that summate to match the measured joint accelerations/moments. The joint angle at which ST slack angle occurred, coupled with the interindividual variability, suggests accurate muscle force estimates may require subject-specific muscle measures and adjusting the passive force-length curve, as done by Lloyd and Besier (71), to allow passive force generation below optimal fiber length. More complex models (e.g., finite element methods) may be needed to assess localized and compartment stresses and strains within ST, though this was not the target of the current study and should be investigated in future studies.

\section{Methodological Considerations and Study Limitations}

The piecewise model was accepted for only $79 \%$ of the tested ST compartments. Data not accepted did not appear to have a plateau region in shear modulus-joint angle curves (see Supplemental data plots) and were generally from both compartments of a given leg. Thus, parameters from compartments with $\theta_{\mathrm{o}} \geq 85^{\circ}$ may indeed be accurate. Conversely, previous studies fitting a piecewise model to 2D-SWE data obtained passively had acquired shear moduli at $\leq 5^{\circ}$ intervals and with a relatively larger plateau (slack) region (50, 72). Measuring at $\leq 5^{\circ}$ intervals throughout a greater range of motion for the present study was not time feasible without conducting slow, passive stretches on an isokinetic dynamometer. However, we deemed it necessary to reposition the transducer between joint angles to maintain image location along the muscle (due to substantial sliding under the skin), optimize image quality, and reduce systematic measurement errors. Thus, it is possible there may have been a very narrow plateau region for these compartments had we measured at smaller joint angle intervals. Although we cannot confirm the accuracy of results where $\theta_{0} \geq 85^{\circ}$, we reperformed the linear mixed model including all data with $R^{2} \geq 0.85$. As the main results did not change (Supplemental Fig. S2), we are confident in our overall interpretation.

The following limitations should also be considered when interpreting the findings of the present study. First, although the leg was held manually at each joint angle rather than controlled by an isokinetic dynamometer, our absolute reliability measures were comparable with previous literature and additional analyses accounting for the measurement error did not affect the interpretation (Supplemental Table S1 and Fig. S1). Thus, the limitations of our experimental setup likely did not influence the results or our conclusions. Second, we only obtained passive measurements in the prone position. Previous studies depicting passive ST shear modulus-joint angle curves obtained in $70^{\circ}-120^{\circ}$ of hip flexion demonstrated an almost immediate increase in shear modulus, despite measurements commencing at $110^{\circ}-120^{\circ}$ of knee flexion (see Fig. $5 A$ of Ref. 28 and Fig. 2 of Ref. 29). 
Therefore, placing the hip in its least flexed position was necessary for assessing ST slack angle. Given the $\alpha$ parameter, representing the exponential increase in shear modulus, did not differ between ST compartments, it is unlikely that hip flexion, where muscle length and thus shear modulus are both greater, would influence the findings. It should also be noted that the optimal sarcomere length of ST (as measured passively) is found within the prone range of knee motion (18, 43,65 ). As a muscle's optimum length is not considered to be a short muscle length and increases in shear modulus were found from the beginning to end of the prone range of motion in the present study (Fig. 3), even when accounting for the measurement error (Supplemental Fig. S1), our experimental design should not be considered insufficient for assessing ST slack angle. Third, images were obtained close to the tendinous inscription and not at different locations within each compartment; however, as the anatomical cross-sectional area of ST varies greatly along its length (47), confining data acquisition to a small region of the whole muscle reduced the effects anatomical cross-sectional area may have on the slope of the increase in the shear modulus-force relationship (22). In addition, image quality can be poor in more proximal portions of $\mathrm{ST}_{\text {prox }}(18,20)$. Fourth, although we ensured electromyography silence of semimembranosus during data acquisition, we cannot definitively confirm ST was inactive as it was not possible to simultaneously acquire electromyograms and elastograms given 1) the large gluteals covering $\mathrm{ST}_{\text {prox }}$ proximal to the transducer, and 2) the available $\mathrm{ST}_{\text {dist }}$ electrode position being in the distal portion of the muscle, where ST is small compared with the adjacent synergists (47) and thus exposing the recordings to high amounts of cross talk. Nevertheless, given shear modulus-joint angle curves did not differ between compartments, any residual activation in ST may support the paradigm of force-sharing between compartments. Finally, the sample size did not allow for male and female subgroups; however, previous work found no between-sex differences in passive ST shear modulus (26) or slack angle of other lower limb muscles $(50,73)$.

\section{Conclusions}

The passive stretching response did not differ between the anatomically in-series proximal and distal compartments of the human ST muscle. In addition, the experimentally determined slack angle occurred at larger knee flexion angles than slack angle estimates from commonly used musculoskeletal models. The overall findings of this study suggest the passive mechanical properties of proximal and distal ST compartments are not different and may be used to improve musculoskeletal model estimates of ST muscle force.

\section{SUPPLEMENTAL DATA}

Supplemental Table S1 and Supplemental Figs. S1 and S2: https://doi.org/10.6084/m9.figshare.16837417.

\section{ACKNOWLEDGMENTS}

The authors thank Dr. Petri Tanska for assistance with MATLAB coding of the piecewise exponential model, Prof. Heikki O. Tikkanen for assistance with obtaining ethical approval for this study, and the HUMEA laboratory (University of Eastern Finland) for providing the electromyography and goniometer equipment.

\section{GRANTS}

This work was supported in part by a Griffith University Postgraduate Research Scholarship and International Experience Incentive Scheme travel grant, the International Society of Biomechanics International Travel Grant program, the Erasmus+ Programme of the European Union, and the Academy of Finland (Grant No. 324529 and 332915).

\section{DISCLOSURES}

No conflicts of interest, financial or otherwise, are declared by the authors.

\section{AUTHOR CONTRIBUTIONS}

A.K., D.J.S., R.S.B., and L.S. conceived and designed research; A.K., K.J.L., and L.S. performed experiments; A.K. analyzed data; A.K., D.J.S., R.S.B., R.K.K., and L.S. interpreted results of experiments; A.K. prepared figures; A.K. drafted manuscript; A.K., D.J.S., K.J.L., R.S.B., H.K., O.L., L.E.D., R.K.K., and L.S. edited and revised manuscript; A.K., D.J.S., K.J.L., R.S.B., H.K., O.L., L.E.D., R.K.K., and L.S. approved final version of manuscript.

\section{REFERENCES}

1. Woodley SJ, Mercer SR. Hamstring muscles: architecture and innervation. Cells Tissues Organs 179: 125-141, 2005. doi:10.1159/ 000085004.

2. Lee TC, O'Driscoll KJ, McGettigan P, Moraes D, Ramphall S, O'Brien M. The site of the tendinous interruption in semitendinosus in man. J Anat 157: 229-231, 1988.

3. van der Made AD, Wieldraaijer T, Kerkhoffs GM, Kleipool RP, Engebretsen L, van Dijk CN, Golanó P. The hamstring muscle complex. Knee Surg Sports Traumatol Arthrosc 23: 2115-2122, 2015. doi:10.1007/s00167-013-2744-0.

4. Edgerton VR, Bodine SC, Roy RR. Muscle architecture and performance: stress and strain relationships in a muscle with two compartments arranged in series. In: Muscular Function in Exercise and Training. 3rd International Symposium on Biological Sciences in Sport, Nice, October/November 1986, edited by Marconnet P, Komi PV. Basel: Karger, 1987, p. 12-23. doi:10.1159/000414703.

5. Haines RW. The homologies of the flexor and adductor muscles of the thigh. J Morpho/ 56: 21-49, 1934. doi:10.1002/jmor.1050560103.

6. Lance-Jones $\mathbf{C}$. The morphogenesis of the thigh of the mouse with special reference to tetrapod muscle homologies. J Morphol 162: 275-309, 1979. doi:10.1002/jmor.1051620207.

7. Trotter JA, Richmond FJR, Purslow PP. Functional morphology and motor control of series-fibered muscles. Exerc Sport Sci Rev 23: 167-213, 1995. doi:10.1249/00003677-199500230-00008.

8. Kellis E, Galanis N, Natsis K, Kapetanos G. In vivo and in vitro examination of the tendinous inscription of the human semitendinosus muscle. Cells Tissues Organs 195: 365-376, 2012. doi:10.1159/ 000327574.

9. Bodine SC, Roy RR, Meadows DA, Zernicke RF, Sacks RD, Fournier M, Edgerton VR. Architectural, histochemical, and contractile characteristics of a unique biarticular muscle: the cat semitendinosus. J Neurophysiol 48: 192-201, 1982. doi:10.1152/jn.1982.48.1.192.

10. Chanaud CM, Pratt CA, Loeb GE. Functionally complex muscles of the cat hindlimb. $\mathrm{V}$. The roles of histochemical fiber-type regionalization and mechanical heterogeneity in differential muscle activation. Exp Brain Res 85: 300-313, 1991. doi:10.1007/BF00229408.

11. Persad LS, Binder-Markey BI, Shin AY, Kaufman KR, Lieber RL. In vivo human gracilis whole-muscle passive stress-sarcomere strain relationship. J Exp Biol 224: jeb242722, 2021. doi:10.1242/jeb. 242722. 
12. Zwambag DP, Gsell KY, Brown SHM. Characterization of the passive mechanical properties of spine muscles across species. $J$ Biomech 88: 173-179, 2019. doi:10.1016/j.jbiomech.2019.03.036.

13. Meyer G, Lieber RL. Muscle fibers bear a larger fraction of passive muscle tension in frogs compared with mice. J Exp Biol 221: jeb. 182089, 2018. doi:10.1242/jeb.182089.

14. Smith LR, Lee KS, Ward SR, Chambers HG, Lieber RL. Hamstring contractures in children with spastic cerebral palsy result from a stiffer extracellular matrix and increased in vivo sarcomere length. $J$ Physio/ 589: 2625-2639, 2011. doi:10.1113/jphysiol.2010.203364.

15. Bigland-Ritchie B, Fuglevand AJ, Thomas CK. Contractile properties of human motor units: is man a cat? Neuroscientist 4: 240-249, 1998. doi:10.1177/107385849800400413.

16. Cronin NJ, Prilutsky BI, Lichtwark GA, Maas H. Does ankle joint power reflect type of muscle action of soleus and gastrocnemius during walking in cats and humans? J Biomech 46: 1383-1386, 2013. doi:10.1016/j.jbiomech.2013.02.023.

17. Kubota J, Kaneko F, Shimada M, Torii S, Fukubayashi T. Effect of joint position on the electromyographic activity of the semitendinosus muscle. Electromyogr Clin Neurophysiol 49: 149-154, 2009.

18. Haberfehlner H, Maas H, Harlaar J, Becher JG, Buizer Al, Jaspers RT. Freehand three-dimensional ultrasound to assess semitendinosus muscle morphology. J Anat 229: 591-599, 2016. doi:10.1111/joa. 12501.

19. Haberfehlner H, Jaspers RT, Rutz E, Becher JG, Harlaar J, van der Sluijs JA, Witbreuk MM, Romkes J, Freslier M, Brunner R, Maas H, Buizer Al. Knee moment-angle characteristics and semitendinosus muscle morphology in children with spastic paresis selected for medial hamstring lengthening. PLoS One 11: e0166401, 2016. doi:10.1371/journal.pone.0166401.

20. Hanssen B, De Beukelaer N, Schless S-H, Cenni F, Bar-On L, Peeters N, Molenaers G, Van Campenhout A, Van den Broeck C, Desloovere K. Reliability of processing 3-D freehand ultrasound data to define muscle volume and echo-intensity in pediatric lower limb muscles with typical development or with spasticity. Ultrasound Med Biol 47: 2702-2712, 2021. doi:10.1016/j.ultrasmedbio.2021.04.028.

21. Hug F, Tucker K, Gennisson J-L, Tanter M, Nordez A. Elastography for muscle biomechanics: toward the estimation of individual muscle force. Exerc Sport Sci Rev 43: 125-133, 2015. doi:10.1249/JES. 0000000000000049 .

22. Koo TK, Hug F. Factors that influence muscle shear modulus during passive stretch. J Biomech 48: 3539-3542, 2015. doi:10.1016/j. jbiomech.2015.05.038.

23. Mendes B, Firmino T, Oliveira R, Neto T, Infante J, Vaz JR, Freitas SR. Hamstring stiffness pattern during contraction in healthy individuals: analysis by ultrasound-based shear wave elastography. Eur $J$ Appl Physio/ 118: 2403-2415, 2018. doi:10.1007/s00421-018-3967-z.

24. Evangelidis PE, Shan $X$, Otsuka S, Yang C, Yamagishi T, Kawakami Y. Hamstrings load bearing in different contraction types and intensities: a shear-wave and B-mode ultrasonographic study. PLoS One 16: e0251939, 2021. doi:10.1371/journal.pone.0251939.

25. Morales-Artacho AJ, Lacourpaille L, Guilhem G. Effects of warm-up on hamstring muscles stiffness: cycling vs foam rolling. Scand J Med Sci Sports 27: 1959-1969, 2017. doi:10.1111/sms.12832.

26. Avrillon S, Lacourpaille L, Hug F, Le Sant G, Frey A, Nordez A, Guilhem G. Hamstring muscle elasticity differs in specialized highperformance athletes. Scand J Med Sci Sports 30: 83-91, 2020. doi:10.1111/sms.13564.

27. Miyamoto N, Kimura N, Hirata K. Non-uniform distribution of passive muscle stiffness within hamstring. Scand J Med Sci Sports 30: 1729-1738, 2020. doi:10.1111/sms.13732.

28. Le Sant G, Ates F, Brasseur J-L, Nordez A. Elastography study of hamstring behaviors during passive stretching. PLoS One 10: e0139272, 2015. doi:10.1371/journal.pone.0139272.

29. Miyamoto N, Hirata K, Kanehisa H. Effects of hamstring stretching on passive muscle stiffness vary between hip flexion and knee extension maneuvers. Scand J Med Sci Sports 27: 99-106, 2017. doi:10.1111/sms.12620.

30. Komi PV, Fukashiro S, Järvinen M. Biomechanical loading of Achilles tendon during normal locomotion. Clin Sports Med 11: 521 531, 1992. doi:10.1016/S0278-5919(20)30506-8.

31. Zajac FE. Muscle and tendon: properties, models, scaling, and application to biomechanics and motor control. Crit Rev Biomed Eng 17: 359-411, 1989.
32. Millard M, Uchida T, Seth A, Delp SL. Flexing computational muscle: modeling and simulation of musculotendon dynamics. J Biomech Eng 135: 021005, 2013. doi:10.1115/1.4023390.

33. Thelen DG. Adjustment of muscle mechanics model parameters to simulate dynamic contractions in older adults. J Biomech Eng 125: 70-77, 2003. doi:10.1115/1.1531112.

34. Delp SL, Loan JP, Hoy MG, Zajac FE, Topp EL, Rosen JM. An interactive graphics-based model of the lower extremity to study orthopaedic surgical procedures. IEEE Trans Biomed Eng 37: 757-767, 1990. doi:10.1109/10.102791.

35. Winters TM, Takahashi M, Lieber RL, Ward SR. Whole muscle length-tension relationships are accurately modeled as scaled sarcomeres in rabbit hindlimb muscles. J Biomech 44: 109-115, 2011. doi:10.1016/j.jbiomech.2010.08.033.

36. Brown IE, Liinamaa TL, Loeb GE. Relationships between range of motion, lo, and passive force in five strap-like muscles of the feline hind limb. J Morphol 230: 69-77, 1996. doi:10.1002/(SICI)1097-4687 (199610)230:1<69::AID-JMOR6>3.0.CO;2-I.

37. Gareis H, Solomonow M, Baratta R, Best R, D'Ambrosia R. The isometric length-force models of nine different skeletal muscles. J Biomech 25: 903-916, 1992. doi:10.1016/0021-9290(92)90230-x.

38. Yucesoy CA, Koopman B, Baan GC, Grootenboer HJ, Huijing PA. Extramuscular myofascial force transmission: experiments and finite element modeling. Arch Physiol Biochem 111: 377-388, 2003. doi:10.3109/13813450312331337630

39. Paudyal A, Degens H, Baan GC, Noort W, Slevin M, van Wegen E, Kwakkel G, Maas H. Changes in muscle-tendon unit length-force characteristics following experimentally induced photothrombotic stroke cannot be explained by changes in muscle belly structure. Eur J Appl Physiol 121: 2509-2519, 2021. doi:10.1007/s00421-02104729-X.

40. Van Der Krogt MM, Bar-On L, Kindt T, Desloovere K, Harlaar J. Neuro-musculoskeletal simulation of instrumented contracture and spasticity assessment in children with cerebral palsy. J Neuroeng Rehabil 13: 64, 2016. doi:10.1186/s12984-016-0170-5.

41. Harlaar J, Becher JG, Snijders CJ, Lankhorst GJ. Passive stiffness characteristics of ankle plantar flexors in hemiplegia. Clin Biomech (Bristol, Avon) 15: 261-270, 2000. doi:10.1016/s0268-0033(99) 00069-8.

42. Rajagopal A, Dembia CL, DeMers MS, Delp DD, Hicks JL, Delp SL. Full body musculoskeletal model for muscle-driven simulation of human gait. IEEE Trans Biomed Eng 63: 2068-2079, 2016. doi:10.1109/TBME.2016.2586891.

43. Ward SR, Eng CM, Smallwood LH, Lieber RL. Are current measurements of lower extremity muscle architecture accurate? Clin Orthop Relat Res 467: 1074-1082, 2009. doi:10.1007/s11999-008-0594-8.

44. Wickiewicz TL, Roy RR, Powell PL, Edgerton VR. Muscle architecture of the human lower limb. Clin Orthop Relat Res 179: 275-283, 1983. doi:10.1097/00003086-198310000-00042.

45. Williams GN, Snyder-Mackler L, Barrance PJ, Axe MJ, Buchanan TS. Muscle and tendon morphology after reconstruction of the anterior cruciate ligament with autologous semitendinosus-gracilis graft. J Bone Joint Surg Am 86: 1936-1946, 2004. doi:10.2106/00004623200409000-00012.

46. Morris N, Jordan MJ, Sumar S, Adrichem B, Heard M, Herzog W. Joint angle-specific impairments in rate of force development, strength, and muscle morphology after hamstring autograft. Trans/ Sports Med 4: 104-114, 2021. doi:10.1002/tsm2.189.

47. Kositsky A, Gonçalves BAM, Stenroth L, Barrett RS, Diamond LE, Saxby DJ. Reliability and validity of ultrasonography for measurement of hamstring muscle and tendon cross-sectional area. Ultrasound Med Biol 46: 55-63, 2020. doi:10.1016/j.ultrasmedbio.2019.09.013.

48. Shiina T, Nightingale KR, Palmeri ML, Hall TJ, Bamber JC, Barr RG, Castera L, Choi BI, Chou Y-H, Cosgrove D, Dietrich CF, Ding H, Amy D, Farrokh A, Ferraioli G, Filice C, Friedrich-Rust M, Nakashima K, Schafer F, Sporea I, Suzuki S, Wilson S, Kudo M. WFUMB guidelines and recommendations for clinical use of ultrasound elastography: Part 1: basic principles and terminology. Ultrasound Med Biol 41: 1126-1147, 2015. doi:10.1016/j.ultrasmedbio. 2015.03.009.

49. Creze M, Nordez A, Soubeyrand M, Rocher L, Maître X, Bellin MF. Shear wave sonoelastography of skeletal muscle: basic principles, biomechanical concepts, clinical applications, and future 
perspectives. Skeletal Radiol 47: 457-471, 2018. doi:10.1007/s00256017-2843-y.

50. Koo TK, Guo JY, Cohen JH, Parker KJ. Quantifying the passive stretching response of human tibialis anterior muscle using shear wave elastography. Clin Biomech (Bristol, Avon) 29: 33-39, 2014. doi:10.1016/j.clinbiomech.2013.11.009.

51. Xu J, Hug F, Fu SN. Stiffness of individual quadriceps muscle assessed using ultrasound shear wave elastography during passive stretching. J Sport Health Sci 7: 245-249, 2018. doi:10.1016/j. jshs.2016.07.001.

52. Modenese L, Ceseracciu E, Reggiani M, Lloyd DG. Estimation of musculotendon parameters for scaled and subject specific musculoskeletal models using an optimization technique. J Biomech 49: 141148, 2016. doi:10.1016/j.jbiomech.2015.11.006.

53. Lacourpaille L, Hug F, Nordez A. Influence of passive muscle tension on electromechanical delay in humans. PLoS One 8: e53159, 2013. doi:10.1371/journal.pone.0053159.

54. Le Sant G, Nordez A, Andrade R, Hug F, Freitas S, Gross R. Stiffness mapping of lower leg muscles during passive dorsiflexion. J Anat 230: 639-650, 2017. doi:10.1111/joa.12589.

55. Bolsterlee B, Finni T, D'Souza A, Eguchi J, Clarke EC, Herbert RD. Three-dimensional architecture of the whole human soleus muscle in vivo. PeerJ 6: e4610, 2018. doi:10.7717/peerj.4610.

56. Maas H. Significance of epimuscular myofascial force transmission under passive muscle conditions. J Appl Physiol (1985) 126: 14651473, 2019. doi:10.1152/japplphysiol.00631.2018.

57. Moo EK, Fortuna R, Sibole SC, Abusara Z, Herzog W. In vivo sarcomere lengths and sarcomere elongations are not uniform across an intact muscle. Front Physiol 7: 187, 2016. doi:10.3389/fphys.2016. 00187.

58. Hodson-Tole EF, Wakeling JM, Dick TJM. Passive muscle-tendon unit gearing is joint dependent in human medial gastrocnemius. Front Physiol 7: 95, 2016. doi:10.3389/fphys.2016.00095.

59. Frich LH, Lambertsen KL, Hjarbaek J, Dahl JS, Holsgaard-Larsen A. Musculoskeletal application and validation of speckle-tracking ultrasonography. BMC Musculoskelet Disord 20: 192, 2019. doi:10.1186/s12891-019-2562-8.

60. Gijsbertse K, Sprengers AMJ, Nillesen MM, Hansen HHG, Lopata RGP, Verdonschot N, de Korte CL. Three-dimensional ultrasound strain imaging of skeletal muscles. Phys Med Biol 62: 596-611, 2017. doi:10.1088/1361-6560/aa5077.

61. Lichtwark GA, Farris DJ, Chen X, Hodges PW, Delp SL. Microendoscopy reveals positive correlation in multiscale length changes and variable sarcomere lengths across different regions of human muscle. J Appl Physiol (1985) 125: 1812-1820, 2018. doi:10.1152/japplphysiol.00480.2018.

62. Stubbs PW, Walsh LD, D'Souza A, Héroux ME, Bolsterlee B Gandevia SC, Herbert RD. History-dependence of muscle slack length following contraction and stretch in the human vastus lateralis. J Physio/ 596: 2121-2129, 2018. doi:10.1113/JP275527.

63. Kaya CS, Bilgili F, Akalan NE, Yucesoy CA. Intraoperative testing of passive and active state mechanics of spastic semitendinosus in conditions involving intermuscular mechanical interactions and gait relevant joint positions. J Biomech 103: 109755, 2020. doi:10.1016/j. jbiomech.2020.109755.

64. Persad LS, Ates F, Shin AY, Lieber RL, Kaufman KR. Measuring and modeling in vivo human gracilis muscle-tendon unit length. J Biomech 125: 110592, 2021. doi:10.1016/j.jbiomech.2021.110592.

65. Cutts A. The range of sarcomere lengths in the muscles of the human lower limb. J Anat 160: 79-88, 1988.

66. Ward SR, Winters TM, O'Connor SM, Lieber RL. Non-linear scaling of passive mechanical properties in fibers, bundles, fascicles and whole rabbit muscles. Front Physiol 11: 211, 2020. doi:10.3389/ fphys.2020.00211.

67. Lieber RL, Binder-Markey BI. Biochemical and structural basis of the passive mechanical properties of whole skeletal muscle. $J$ Physio/ 599: 3809-3823, 2021. doi:10.1113/JP280867.

68. Moo EK, Leonard TR, Herzog W. The sarcomere force-length relationship in an intact muscle-tendon unit. J Exp Bio/ 223: jeb215020, 2020. doi:10.1242/jeb.215020.

69. Schache AG, Dorn TW, Blanch PD, Brown NAT, Pandy MG. Mechanics of the human hamstring muscles during sprinting. Med Sci Sports Exerc 44: 647-658, 2012. doi:10.1249/MSS.0b013e318236a3d2.

70. Chumanov ES, Heiderscheit BC, Thelen DG. The effect of speed and influence of individual muscles on hamstring mechanics during the swing phase of sprinting. J Biomech 40: 3555-3562, 2007. doi:10.1016/j.jbiomech.2007.05.026.

71. Lloyd DG, Besier TF. An EMG-driven musculoskeletal model to estimate muscle forces and knee joint moments in vivo. J Biomech 36: 765-776, 2003. doi:10.1016/S0021-9290(03)00010-1.

72. Maïsetti O, Hug F, Bouillard K, Nordez A. Characterization of passive elastic properties of the human medial gastrocnemius muscle belly using supersonic shear imaging. J Biomech 45: 978-984, 2012. doi:10.1016/j.jbiomech.2012.01.009.

73. Miyamoto N, Hirata K, Miyamoto-Mikami E, Yasuda O, Kanehisa H. Associations of passive muscle stiffness, muscle stretch tolerance, and muscle slack angle with range of motion: individual and sex differences. Sci Rep 8: 8274, 2018. doi:10.1038/s41598-01826574-3. 